\title{
EDITORIAL
}

\section{Moving clinical research in academic medical centres up the value chain}

\author{
A decline in the approval of new molecular entities - particularly truly innovative ones - \\ has coincided with an increasing emphasis on translational medicine by funders of the \\ academic sector. How might the full potential of academic medical centres be realized?
}

By 2012, the US National Institutes of Health plans to invest $\sim$ US\$500 million in Clinical and Translational Science Awards (CTSAs) in $\sim 60$ academic medical centres (AMCs). Among the objectives of the CTSAs, which have so far been awarded to 39 AMCs, has been the enhancement of infrastructure to facilitate the translation of scientific discovery into clinical benefit.

A review of the obstacles that retard the initiation of clinical research in AMCs has been an intrinsic part of this effort. Much of the initial focus is on how efficient AMCs are in comparison with community physicians in the United States regarding the initiation of and recruitment for multicentre, industry-sponsored Phase III trials.

The general picture is depressing. Compared with community-based physician practices and contract research organizations (CROs), AMCs are slow to approve such studies, initiate recruitment and meet recruitment goals. Indeed, many studies that are initiated in AMCs fail to recruit a single patient.

So, why do AMCs perform so poorly in this respect and what can be done about it? Apart from deficiencies in the process - delays in ethical and scientific review and in formulating contracts - that could be overcome by prioritizing investment in time, people and money, there is a basic misalignment of incentives for this type of research within most AMCs ${ }^{1}$. Although considerable expertise in the design, organization and conduct of industry-sponsored Phase III trials resides in a minority of AMCs, elsewhere such studies, with a preset protocol, have limited relevance to promotion to tenure and usually involve faculty members whose main responsibility is the provision of clinical care. Furthermore, the financial incentive of efficiency - directly relevant to CROs and community physicians - is not a key factor in an AMC, as clinical faculty are salaried employees.

A metric commonly used by sponsors of such trials is the cost per patient completing the trial. It is difficult for AMCs, with their broad and diverse missions, to compete with CROs, for which the core business is such trials, let alone with foreign sites that have low cost structures. So, should substantial effort be invested in building capacity in AMCs to perform this type of clinical research? Perhaps.
There is, however, a more valuable alternative, related to the growing recognition that the traditional approach to drug development, dominated by large, fully integrated pharmaceutical companies, is unsustainable ${ }^{2,3}$. Industry has traditionally focused on the rapid transition from initial evidence of tolerability in humans to the selection of dose and initiation of Phase III trials. The paucity of experts in translational therapeutics, coupled with pressure from marketing departments, has led to a tendency to accelerate drug development past Phase II trials with an incomplete understanding of drug action. The legacy of this strategy is a decrease in the number of approved novel drugs and unanticipated adverse events post-approval.

A shift towards a more flexible and modular approach that engages both industry and academia therefore seems likely. As this occurs, attempts to personalize medicine will move emphasis on to Phase II trials. Here, the detailed elucidation of mechanisms of drug action and the factors that determine variability of drug response in humans will require complex phenotyping approaches that are beyond the capability of typical community physicians or CROs. Such studies have the capacity for a high academic content, relevant both to publication and the promotion of faculty members involved.

In this case, the incentives are aligned to engage tenure-track faculty in the timely pursuit of clinical research. Most leading AMCs might usefully build 'human capital' in translational therapeutics and aim to facilitate single-site, highly complex studies of drug action in relatively small numbers of people. In time, as a consortium of such centres is established, these complex studies could be expanded. Achieving this goal requires novel educational initiatives, the branding and support of this emerging discipline, expansion of infrastructure in informatics and appropriate research facilities. Such a qualitative shift in the type of clinical research would align strategy with the capabilities that are unique to AMCs and could prove the key to realizing their potential. ThA Consortium Steering Committee, but the opinions expressed here are his own.

e-mail:garret@upenn.edu
Garret A. FitzGerald
1. von Herrath, M. \& Chan, A. J. Clin. Invest.119, 1061-1065 (2009). 2. FitzGerald, G. A. Science 320, 1563 (2008).
3. Dixon, J., Lawton, G. \& Machin, P. Nature Rev. Drug Discov. 8, 435 (2009).

